\title{
Parametric Model Method and Deployment Simulation of Inflatable Antenna Structures
}

\author{
Xu Yan¹, Zheng Yao', Guan Fuling², Huang He², Xu Xian²
}

\begin{abstract}
In this paper, we develop a parametric model method and simulate the deployment mechanism of inflatable antenna structures. Different folded methods are developed for the primary members of inflatable antenna structures, which include inflatable tubes, an inflatable torus, a reflector etc. The unstressed configuration and the folded configuration of these members are modeled parametrically using the developed folded methods. A simulation software is developed for the deployment mechanism of inflatable structures by the improved spring-mass system. The driving forces in the deployment process, i.e. the gas pressure and the moment of the fold hinges, are analyzed for each member. During the development process, self-contact or collision with the membrane occurs. A rule for identifying self-contact elements is applied, and a penalty function method is developed to solve this challenging problem. Finally, the equation of motion is solved using finite difference method. The developed simulation software is validated by simulating a cylindrical inflatable tube that is folded in half, and the simulation agrees well with the experiment. The deployment mechanism of the antenna model similar to that for the Inflatable Antenna Experiment (IAE) is modeled, analyzed and estimated. The deployed configurations and the dynamic parameters of each node are obtained. The numerical simulation results show that the simulation software for the deployment mechanism can correctly predict the deployment process of inflatable antenna structures.
\end{abstract}

KEYWORDS: Inflatable antenna, Improved spring-mass system, Folded methods, Deployment mechanism simulation.

\section{INTRODUCTION}

Large-size aerospace structures are difficult to construct because of the limitations of their weight and launch volume. Compared to conventional electromechanical deployable structures (Xu et al., 2012), the inflatable structures offer many advantages including design flexibility, lightness in weight and compact launch volumes and are suitable for large-size aerospace structures. Since the 1990s, inflatable technologies have been used for antenna structures in NASA's Jet Propulsion Laboratory etc. Many ideas, concepts and models have been proposed, from the inflatable antenna (Cliff and Ron, 2001; David, 2003) to the inflatable aerodynamic decelerator (Kramer et al., 2013) and the lunar habitat (Capua et al., 2011). One of the key technologies of these structures is that the system can be folded into a compact launch volume and deployed in a smooth and orderly manner. Numerical simulations are needed to investigate the deployment mechanism in greater detail.

Deployment simulations and research studies of inflatable structures to date have primarily focused on inflatable tubes. Simulations have been based on two methods: multi-rigid-body dynamics theory and the non-linear finite element method. In the first simulation method, an inflatable membrane tube is modeled as several rigid struts or beam elements, and the deployment process of the tube can then readily be analyzed using multi-body dynamics theory (Fang et al., 2006). A tube between a two-fold point as inflatable cantilever beams was modeled (Clem and 
Smith, 2000), which was then analyzed using multi-rigidbody dynamic theory. The 3-D deployment mechanism of the inflatable structures must be analyzed using the nonlinear finite element method (Wang and Johnson, 2002; Lampani and Gaudenzi, 2010) and some commercially available software was applied to this simulation work. PAM-CRASH software was used to simulate the deployment of an inflatable structure (Haug et al., 1991; Katsumata et al., 2014). LS-DYNA software was used to analyze the deployment of a tube and accounted for the reciprocity between the gas flow and the flexible tube shell (Salama et al., 2001). The numerical and experimental investigations on the deployment of inflatable tubes with one fold point were conducted (Miyazaki and Uchiki, 2002; Bouzidi et al., 2013). Also the deployment of an inflatable tube was validated by space experiment (Wei et al., 2015). However, only a few research studies have been performed on the deployment mechanism of complicated inflatable structures, which should be investigated further.

For complicated inflatable structures — such as an inflatable antenna, which consists of inflatable tubes, an inflatable torus and a reflector - , it is difficult to model the folded configuration of the entire structure. In this paper, we report on recent efforts to develop a parametric model and deployment simulation software for inflatable antenna structures. The entire antenna structure is modeled parametrically and analyzed, and the deployment mechanism is simulated using an improved spring-mass system in which the self-contact of the membrane is solved using a penalty function method.

\section{FOLDED METHODS AND PARAMETRIC MODEL}

There are four configurations in the deployable simulation of inflatable structures: the unstressed configuration (prior to folding), the initial folded configuration, the configurations during deployment and the final fully deployed configuration. The purpose of the deployment simulation is to simulate the motion from the initial folded configuration to the fully deployed configuration using the known unstressed and the initial folded configurations. In this section, the unstressed and initial folded configurations of the primary members, such as the inflatable tubes, the inflatable torus and the reflector, are designed and modeled parametrically.

\section{INFLATABLE TUBES}

The unstressed configuration of the inflatable tubes is a cylinder shell. The length of the cylinder shell is $L_{1}$ and the section radius is $R_{1}$. The unstressed configuration of the section is a circle, and the folded configuration of the section is shown in Fig. 1, which includes two sections of circular curves. The radius of two circular curves is $R_{2}$ and the corresponding central angle is $\theta$. The strain of the membrane material during the folding process is neglected, which results in an invariant circumference for the tube section. So, the central angle $\theta_{1}$ in the folded configuration can be expressed as follows:

$$
\theta_{1}=\left(\pi R_{1}\right) / R_{2}
$$

The coordination of each node in the circular curves can then be calculated. There are typically two folded patterns for inflatable tubes along the length direction: the curve folded pattern and the zigzag folded pattern. The second folded pattern is used for the inflatable antenna structures (Katsumata et al., 2014).

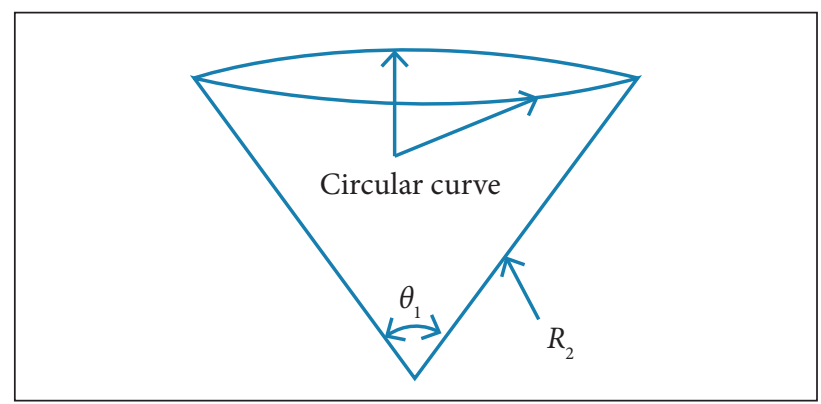

Figure 1. Folded configuration of the tube's section.

\section{INFLATABLE TORUS}

The unstressed configuration of the inflatable torus is a circular shell surface. This surface has a non-zero Gaussian curvature, which causes an initial stress to appear in the membrane after the folding process. Similarly to the inflatable tubes, the unstressed configuration of a section of a torus is a circle, which can be folded in the same way as for the inflatable tubes.

The axes curve of the inflatable torus is also a circle and the radius of the axes curve is denoted by $R$. The developed folded method is shown in Fig. 2a. A circular curve can be approximated by an equilateral polygon. The number of sides of the equilateral polygon is denoted by $n$. The length of each side of the polygon is given as follows:

$$
L=2 \pi R / n
$$


The projected shape in the $0 \mathrm{XY}$ plane is also a polygon after the inflatable torus is folded. $\theta$ is the folded angle of each polygon side along vertical direction. The length of each side is given as follows:

$$
L_{x y}=(2 \pi R / n) \cos \theta
$$

The circumradius of the polygon in the folded configuration is:

$$
r=L_{x y}(R / L)
$$

The projected height of the folded configuration onto the $0 \mathrm{XZ}$ plane is:

$L_{x y}=(2 \pi R / n) \sin \theta$

Thus, the coordination of each fold point is:

$$
x_{i}=r \cos \varphi_{i}, y_{i}=r \sin \varphi_{i}, z_{i}= \pm L_{x z} / 2
$$

where:

$\varphi$ : the corresponding central angle, and the sign of the Z-coordinate alternates.

The folded methods for the axes curve are used for the torus section to calculate the coordination of each node in the folded configuration. The folded configuration of the inflatable torus is shown in Fig. 2b.

\section{INFLATABLE REFLECTOR}

The inflatable reflector consists of a reflective surface and a canopy. The reflective surface assumes a parabolic shape under the inflation pressure. A parabolic surface is difficult to fold. The parabolic surface is a surface of revolution; thus, the surface is first folded along the longitudinal direction and then along the circumferential direction. The fold pattern along the latitudinal direction is similar to that along the axes direction of the inflatable torus. The fold pattern along the latitudinal direction is similar to that of the Z-folded method for inflatable tubes.

The fold pattern of the canopy is similar to that of the reflector surface. First, the canopy is placed near the reflector surface. The Z-coordination of each point on the canopy is $1 \mathrm{~mm}$ smaller than the corresponding point on the reflector surface. The canopy is then folded synchronously with the reflector surface. The parametric models for unstressed configuration and folded configuration of inflatable reflector are shown in Fig. 3.

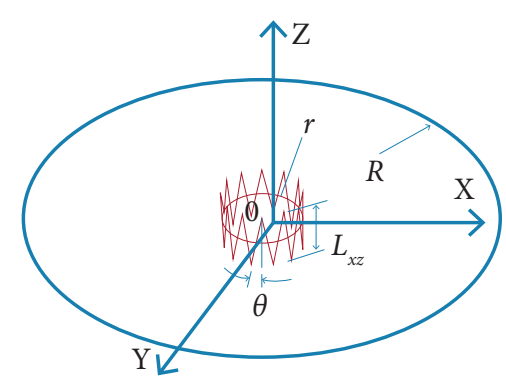

(a)

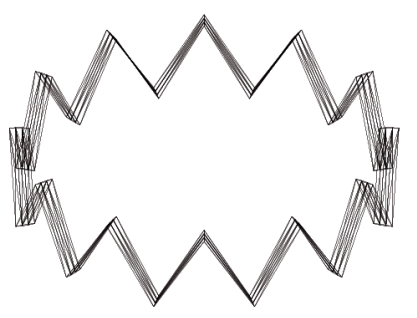

(b)

Figure 2. Parametric model for folded torus. (a) Folded method for axes curve; (b) Folded configuration.

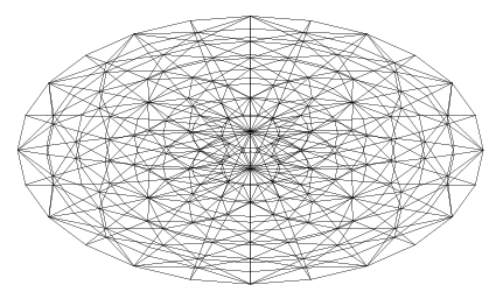

(a)

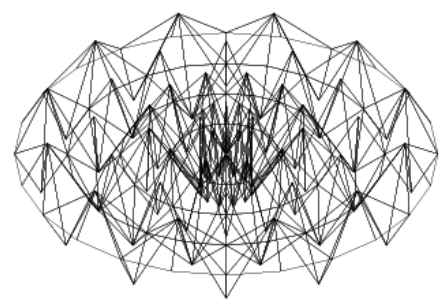

(b)

Figure 3. Parametric models of inflatable reflector. (a) Unstressed configuration; (b) Folded configuration.

\section{INFLATABLE ANTENNA}

A complete model of the inflatable antenna structure, such as that of the Inflatable Antenna Experiment (IAE), includes inflatable tubes, an inflatable torus, a reflector and tension 
cables. The support structure for the inflatable antenna structure consists of three tubes and a torus. There are several tension cables between the reflector and the support structure. Each primary member is folded using the developed method and is assembled to form the initial configuration of the entire structure. The unstressed configuration and the folded configuration of the entire antenna structure are shown in Fig. 4.

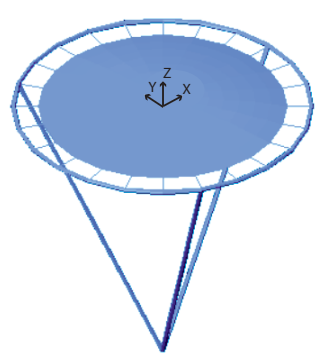

(a)

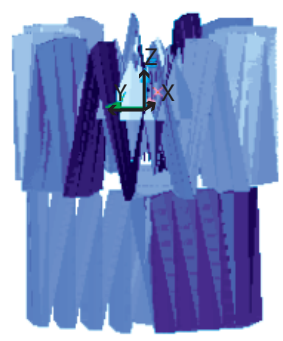

(b)
Figure 4. Parametric models of the entire antenna's structure. (a) Unstressed configuration; (b) Folded configuration.

\section{DEPLOYMENT DYNAMICS SIMULATION METHOD}

\section{DESCRIPTION OF SPRING-MASS SYSTEM}

A spring-mass system is developed to model the membrane material in the deployment simulation. The three vertexes of the triangular membrane element consist of masses, and the three borders consist of springs. The lengths of the springs in their initial state correspond to the original lengths of the springs. During the deployment process, the spring lengths do not equal their original lengths, i.e. internal forces exist in the springs. The three masses move under the combined action of the spring forces and the external forces to reach the final equilibrium configuration. The spring-mass system for an element is shown in Fig. 5.

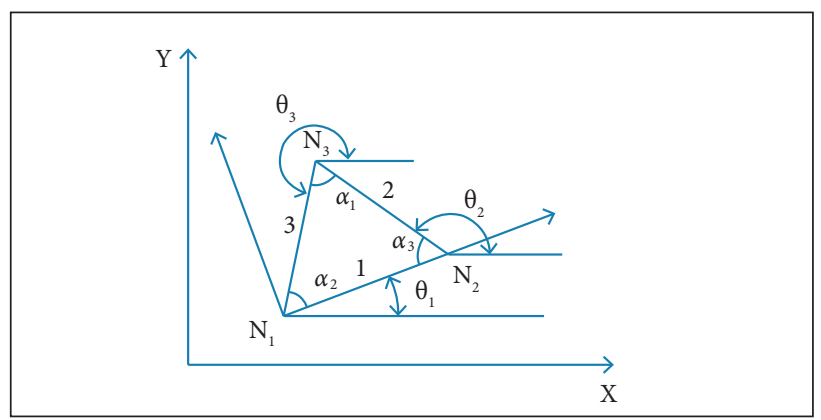

Figure 5. Spring-mass system.
The mass of point $i$ is determined by the area of the membrane elements shown in Fig. 6 and is given as follows:

$$
m_{i}=\frac{\alpha_{i} \cdot \rho}{2 \pi} \sum_{k=1}^{s} A_{k}
$$

where:

$\rho$ : area density of the material; $A_{k}$ : area of element $k$ around the mass point $i$; $s$ number of elements around the mass $i$; $\alpha_{i}$ : internal angle for each vertex of the element.

The tension vector of the springs in the element can be obtained from Xu et al. (2007):

$$
\mathbf{T}=\left\{\begin{array}{l}
T_{1} \\
T_{2} \\
T_{3}
\end{array}\right\}=\mathbf{B}^{T} \mathbf{D B}\left\{\begin{array}{l}
\delta_{1} \\
\delta_{2} \\
\delta_{3}
\end{array}\right\} V
$$

where:

$\delta$ : extension vector of the three springs; $V$ : volume of the triangular element; D: elastic matrix of the membrane material; B: the relationship matrix between the strains of the element and the length deformations of three springs.

$$
\begin{aligned}
& a_{i}=\cos ^{2} \theta_{i}, \mathrm{~b}_{i}=\sin ^{2} \theta_{i}, \mathrm{c}_{i}=\sin \theta_{i} \cos \theta_{i} \\
& \mathbf{A}=\left[\begin{array}{lll}
a_{1} & b_{1} & c_{1} \\
a_{2} & b_{2} & c_{2} \\
a_{3} & b_{3} & c_{3}
\end{array}\right] \\
& \mathbf{D}=\left[\begin{array}{ccc}
\frac{E}{1-v^{2}} & \frac{v E}{1-v^{2}} & 0 \\
\frac{v E}{1-v^{2}} & \frac{E}{1-v^{2}} & 0 \\
0 & 0 & \frac{E}{2(1+v)}
\end{array}\right]
\end{aligned}
$$$$
\mathbf{B}=\frac{1}{|\mathbf{A}|}\left[\begin{array}{lll}
\left(b_{2} c_{3}-b_{3} c_{2}\right) l_{1}^{-1} & \left(b_{3} c_{1}-b_{1} c_{3}\right) l_{2}^{-1} & \left(b_{1} c_{2}-b_{2} c_{1}\right) l_{3}^{-1} \\
\left(a_{3} c_{2}-a_{2} c_{3}\right) l_{1}^{-1} & \left(a_{1} c_{3}-a_{3} c_{1}\right) l_{2}^{-1} & \left(a_{2} c_{1}-a_{1} c_{2}\right) l_{3}^{-1} \\
\left(a_{2} b_{3}-a_{3} b_{2}\right) l_{1}^{-1} & \left(a_{3} b_{1}-a_{1} b_{3}\right) l_{2}^{-1} & \left(a_{1} b_{2}-a_{2} b_{1}\right) l_{3}^{-1}
\end{array}\right]
$$

where:

$E$ : elastic modulus; $v$ : Poisson's ratio; $l_{i}$ initial lengths of the springs; $\theta_{i}$ : angles that the sides of the element make with the $\mathrm{X}$-axis. 


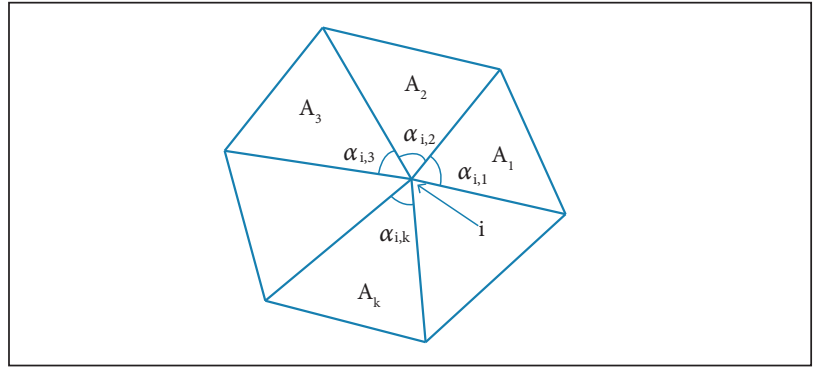

Figure 6. Triangular elements around mass $i$.

\section{DEPLOYMENT DRIVING FORCES}

In this section, the deployment driving forces that lead to the motion of the inflatable primary members are formulated. The driving forces in the inflatable antenna structures have two sources: the gas pressure and the moment of the fold hinge.

The internal chamber of the inflatable tubes and the inflatable torus are divided into a finite number of compartments (Katsumata et al., 2014; Xiao et al., 2010). The inflatable reflector can be considered to be a compartment. The variations in the gas state of each compartment are functions of the deployment time. the temperature and pressure of the inflation gas are also assumed to be uniform within the control volume of an individual compartment. For each compartment, a set of differential governing equations based on gas dynamic theory can be obtained. For three adjacent compartments, $i, j$ and $k$, the differential governing equations for compartment $j$ are given in Eq. 9. For brevity, the formulas are not derived here: further details can be found in Fang et al. (2006).

$$
\begin{aligned}
& {\left[\begin{array}{cccccccc}
0 & 0 & 0 & 0 & m_{j} V_{j} & V_{j} T_{j} & 0 & (k-1) m_{j} T_{j} \\
0 & 0 & 0 & 0 & 0 & 1 & 0 & 0 \\
0 & 0 & 0 & 0 & -m_{j} R & -R T_{j} & V_{j} & P_{j} \\
0 & 0 & V_{j} & 0 & 0 & 0 & -V_{j}^{*} & \left(P_{i}-P_{a}\right)
\end{array}\right]\left\{\begin{array}{c}
\dot{T}_{i} \\
\dot{m}_{i} \\
\dot{P}_{i} \\
\dot{V}_{i} \\
\dot{T}_{j} \\
\dot{m}_{j} \\
\dot{P}_{j} \\
\dot{V}_{j}
\end{array}\right\}=} \\
& \left\{\begin{array}{l}
0 \\
0 \\
0 \\
0 \\
k T_{i} V_{j} \dot{m}_{\text {in }}-k T_{j} V_{j} \dot{m}_{\text {out }} \\
\quad \dot{m}_{\text {in }}-\dot{m}_{\text {out }} \\
0 \\
\quad 0
\end{array}\right\}
\end{aligned}
$$

where:

$T_{i}, m_{i}, P_{i}$, and $V_{i}$ : temperature, mass, pressure and volume of compartment $i ; T_{j}, m_{j}, P_{j}$, and $V_{j}$ : temperature, mass, pressure and volume of compartment $j$; $P_{a}$ : ambient gas pressure; $R=8.3145 \mathrm{~J} / \mathrm{molK} ; k=c_{p} / c_{v}:$ specific heat ratio of the gas; $\dot{m}_{i n}$ : mass rate of the gas flowing into compartment $j$ from compartment $i ; \dot{m}_{\text {out }}$ : mass rate of gas flowing out of compartment $j$ into compartment $k$.

The state of each compartment is then calculated by integrating this group of differential equations with respect to the deployment time. The Runge-Kutta method is used to integrate these equations numerically. The inflation pressure of each compartment is then obtained by performing a gas flow analysis and equals the nodal forces on the mass of the spring-mass system. The nodal forces $\mathbf{F}_{a}^{i}$ of a triangular element a in the shell of compartment $i$ can be expressed in the following form:

$$
\mathbf{F}_{a}^{i}=(1 / 3) A\left\{p_{x} p_{y} p_{z} p_{x} p_{y} p_{z} p_{x} p_{y} p_{z}\right\}
$$

where:

$p_{x}=n_{x} \times P_{i}, p_{y}=n_{y} \times P_{i}, p_{z}=n_{z} \times P_{i} ; P_{i}$ : pressure of compartment $i$; $n_{x}, n_{y}, n_{z}$ : normal vector components of the triangular element plane; $A$ : area of the element.

A non-linear joint model and the Bernoulli-Euler method (Clem and Smith, 2000) were used to obtain the relationship between the restoring moment at a hinge point between two adjacent compartments and the folded angle $\theta$ :

$$
M=\pi p r^{3}[1-\exp (-3.12 E t / p \tan \theta / 2)]
$$

where:

$p$ : inflation pressure of the tube or torus near this fold joint; $r$ : section radius of the member; $E$ and $t$ : Young's modulus and the thickness of the material, respectively.

The restoring moment can easily be transformed into the equivalent nodal forces of the mass point in the springmass system.

\section{SELF-CONTACT PROBLEM}

Membrane collision during the deployment process of the inflatable structures is commonly referred to as the self-contact problem. The self-contact problem is one of the major challenges in a deployment simulation because each thin film moves independently in a 3-D space. One solution for this problem 
is to apply the polygon intersection method, which identifies membrane collision by determining whether the boundary of one membrane film intersects the plane or boundary of another membrane film. If a collision is about to occur at a mass point, the motion of this mass is changed by altering the mass velocity such that the imminent collision is prevented.

In this study, a different method is used to solve the selfcontact problem. To prevent self-contact and collision, a penalty force is added to the moving masses. Provot's method (Wang et al., 2002) is adapted to apply a penalty force when the distance between one mass $A\left(x_{A}, y_{A}, z_{A}\right)$ and a membrane element IJK is less than a fixed value $h_{j}^{*}$ (see Fig. 7). The penalty function is a vector function. When the mass $A$ moves to a position that is too close to the element plane $I J K$, the penalty function is applied at the position of the mass to prevent collision.

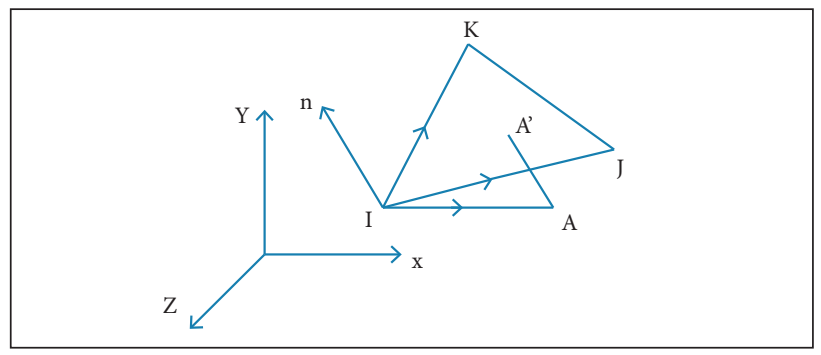

Figure 7. Self-contact element between the mass $A$ and the element IJK.

Given the vectors $\mathbf{X}^{I}, \mathbf{X}^{I}, \mathbf{X}^{K}$ that describe the current position of the nodes in the global coordinate system, the boundary vectors of the triangular element can be defined as follows:

$X^{I I}=X^{I}-X^{I}, X^{K I}=X^{K}-X^{I}, X^{K I}=X^{K}-X^{I}$

The normal vector of the element plane IJK can be defined as follows:

$\mathbf{v}_{3}=\mathbf{X}^{\mathrm{II}} \times \mathbf{X}^{\mathrm{KI}}, \mathbf{n}=\mathbf{v}_{3} /\left\|\mathbf{v}_{3}\right\|=\left(x_{n}, y_{n}, z_{n}\right)$

where:

$n$ : unit normal vector.

The equation of the element plane $I J K$ is given as follows:

$\mathbf{n} \mathbf{x}=\left(\mathbf{X}-\mathbf{X}^{\mathbf{I}}\right)=0$

where:

$\mathrm{X}$ : position vector of a random node in the plane.
First, we determine the side of the plane $I J K$ on which the mass $A$ lies using the following rules:

- if $\mathbf{n} .\left(\mathbf{X}^{\mathrm{A}}-\mathbf{X}^{\mathrm{I}}\right)>0$, the mass $A$ lies on the obverse side of the plane;

- if $\mathbf{n} .\left(\mathbf{X}^{\mathrm{A}}-\mathbf{X}^{\mathrm{I}}\right)<0$, the mass $A$ lies on the reverse side of the plane.

In Fig. 8, the mass $A$ lies on the reverse side of the plane.

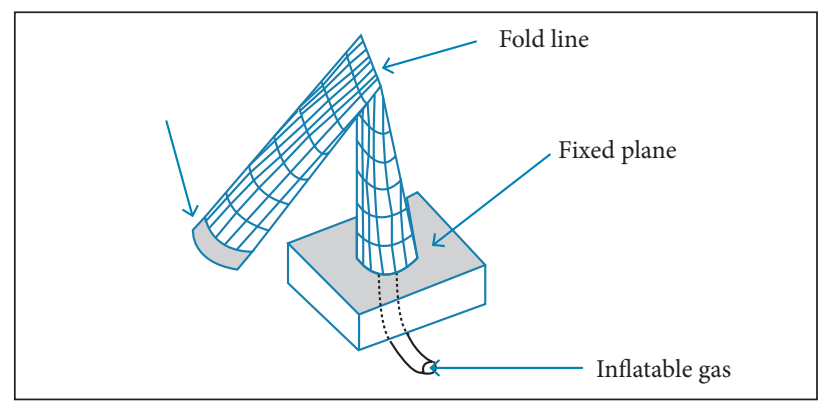

Figure 8. Components of the experimental model.

The projected node $A^{\prime}$ of mass $A$ onto the plane is obtained by solving the following equations:

$$
\left\{\begin{array}{l}
\mathbf{n} \times\left(\mathbf{X}-\mathbf{X}^{\mathbf{I}}\right)=0 \\
\frac{x-x_{A}}{x_{n}}=\frac{y-y_{A}}{y_{n}}=\frac{z-z_{A}}{z_{n}}
\end{array}\right.
$$

We determine whether the projected node $A^{\prime}$ lies in the triangular field by the following method.

A radial line is drawn from node $A^{\prime}$ that is parallel to the $o x$-axis of the local coordinate system. The radial line intersects the boundary of the triangular element. If there is an odd number of intersection points, then the node $A^{\prime}$ lies within the triangular field; otherwise the node $A^{\prime}$ does not lie within the triangular field.

If the projected node $A^{\prime}$ lies within the triangular element field, there is a contact relationship between the mass $A$ and the element $I J K$. When the distance from the mass $A$ to the element $I J K$ is less than the Euclidean distance, a penalty force is added to the mass $A$.

The penalty force for the mass $A$ is defined next.

When the mass $A$ is on the obverse side of the plane $I J K$, the penalty force is given as follows:

$$
\mathbf{F}_{\mathbf{p}}=\sum_{j-1}^{m} C_{p}\left|h_{j}-h_{j}^{*}\right| \mathbf{n}
$$


When the mass $A$ is on the reverse side of the plane $I J K$, the penalty force is given as follows:

$$
\begin{aligned}
\mathbf{F}_{\mathbf{p}}=\sum_{j-1}^{m} C_{p}\left|h_{j}-h_{j}^{*}\right| \mathbf{n} \\
C_{p}= \begin{cases}k_{\text {contact }} & \left(h_{j} \leq h_{j}^{*}\right) \\
0 & \left(h_{j}>h_{j}^{*}\right)\end{cases}
\end{aligned}
$$

where:

$\mathrm{F}_{\mathrm{p}}$ : penalty force vector for the mass $A ; C_{p}$ : penalty coefficient; $k_{\text {contact }}$ interface contact stiffness which depends on the membrane material property; $h_{i}$ : current distance from the mass $A$ to the element plane IJK; $h_{j}^{*}$ : fixed value for the self-contact problem; $n$ : unit normal vector.

If the projected node $A^{\prime}$ does not lie within the triangular element field, there is no contact between the mass $A$ and the element $I J K$.

\section{EQUATION OF MOTION}

The equation of motion for the spring-mass system subjected to internal and external forces is given as follows:

$$
\mathbf{M X}+\mathbf{C} \dot{\mathbf{X}}=\mathbf{F}_{1}+F_{\mathrm{E}}
$$

where:

$\mathbf{M}$ and $\mathbf{C}$ : mass and damping matrices, respectively; $\mathrm{F}_{\mathrm{r}}$ : inertial force arising from the springs; $\mathrm{F}_{\mathrm{E}}$ : generalized external force associated with the degrees of freedom of the model; $\mathbf{x}$ : position vector of all the mass points.

The following equation can be written for each mass:

$$
m \frac{\partial^{2} X}{\partial t^{2}}+c \frac{\partial X}{\partial t}=F_{e x}+F_{\text {in }}
$$

where:

$m$ : mass of each mass point; $c$ : damping of each mass point; $F_{e x}$ inertial force subjected on each mass point; $F_{i n}$ :generalized external force subjected on each mass point.

The finite difference method can be used to simplify the two terms on the left-hand side of Eq. 19:

$$
\begin{aligned}
& m \frac{\partial^{2} X}{\partial t^{2}}=m \frac{1}{\Delta t^{2}}\left(X_{t+\Delta t}-2 X_{t}+X_{t-\Delta t}\right), \\
& c \frac{\partial X}{\partial t}=c \frac{1}{2 \Delta t}\left(X_{t+\Delta t}-X_{t-\Delta t}\right)
\end{aligned}
$$

Substituting Eq. 20 into Eq. 19, one yields:

$$
\begin{aligned}
& m \frac{1}{\Delta t^{2}}\left(X_{t+\Delta t}-2 X_{t}+X_{t-\Delta t}\right)+ \\
& c \frac{1}{2 \Delta t}\left(X_{t+\Delta t}-X_{t-\Delta t}\right)=
\end{aligned}
$$

$$
\begin{aligned}
& F_{e x}+F_{\text {in }}\left(m \frac{1}{\Delta t^{2}}+c \frac{1}{2 \Delta t}\right) X_{t+\Delta t}=F_{e x}+F_{i n}+ \\
& m \frac{2}{\Delta t^{2}} X_{t}-\left(m \frac{1}{\Delta t^{2}}-c \frac{1}{2 \Delta t}\right) X_{t-\Delta t}
\end{aligned}
$$

Let us define the following terms:

$$
M=m \frac{1}{\Delta t^{2}}, C=c \frac{1}{2 \Delta t}, F=F_{e x}+F_{\text {in }}
$$

Then, we can write the following expression for the entire spring-mass system:

$$
(\mathbf{M}+\mathbf{C}) \mathbf{X}_{t+\Delta t}=\mathbf{F}_{e x}+\mathbf{F}_{i n}+2 \mathbf{M X}-(\mathbf{M}-\mathbf{C}) \mathbf{X}_{t-\Delta t}
$$

The initial configuration of the system corresponds to a static state. Thus, the displacement, the velocity and the acceleration at time 0 are zero.

$$
\begin{aligned}
& \left.\mathbf{X}_{t}\right|_{t=0}=0,\left.\mathbf{X}_{t-\Delta t}\right|_{t=0}=0, \\
& \left.\frac{\partial \mathbf{X}_{t}}{\partial t}\right|_{t=0}=0,\left.\frac{\partial^{2} \mathbf{X}_{t}}{\partial t^{2}}\right|_{t=0}=0
\end{aligned}
$$

In the deployment process, the initial configuration is the folded configuration $S_{0}$ of the inflatable structures. The structures are deployed into the configuration $S_{i}$ by a generalized external force. Eq. 8 is used to compute the distortion and the internal force of each spring. A penalty force is added to each of the masses in which self-contact is about to occur. Eqs. 20 and 23 are then used to calculate the values of the velocity, the acceleration and the displacement at this time; these values are added to the configuration $S_{i}$ to obtain the new configuration $S_{i+1}$. 


\section{DEPLOYMENT SIMULATION SCHEME}

A simple computational scheme is in the deployment mechanism simulation. This scheme is summarized next.

- The geometric parameters of the unstressed configuration and the material parameters of membranes and cables are inputted.

- Folded methods are designed, and the initial folded configuration of the entire structure is modeled parametrically.

- The spring-mass system is formulated to describe the membrane material.

- The deployment driving forces are calculated and applied to the spring-mass system.

- The self-contact between each mass and each membrane element is determined, and a penalty force is added to each of the masses at which self-contact is about to occur.

- The boundary conditions are applied, and the equation of motion is solved using the finite difference method.

- The configurations during the deployment process are obtained and saved as an AVI video file.

- The deployment mechanism of the entire process and the structural performance of the fully deployed configuration are evaluated.

The aforementioned simulation scheme is used to formulate the deployment simulation code for the inflatable antenna structures that is compiled in Fortran, and the software interface is compiled in MFC and OpenGL. The software consists of a preprocess module, a solver module and a post-process module. In the pre-process module, the material and geometric parameters are inputted, and the parametric model code is run. The numerical model of the folded configuration is then displayed graphically. After the numerical model of folded configuration is verified, the solver code is called, and the progress of the analysis process is shown. The analysis results can be opened in the post-process module. The formats of the analysis result files are .dxf, .avi and .txt. The deployed configurations and the fully deployed configuration are displayed by the visualization module and are subsequently analyzed to evaluate the deployment mechanism.

\section{NUMERICAL EXAMPLES Z-FOLD PATTERN}

The developed simulation software is validated by simulating a cylindrical inflatable tube that is folded in half: the results are compared to the analysis and experimental results from Miyazaki and Uchiki (2002). Each part of the deployment experimental model for this tube is shown in Fig. 8. The bottom end of the tube is fixed, and a rigid column is attached to the tip of the tube. The initial folded configuration of the model in the simulation is shown in Fig. 9.

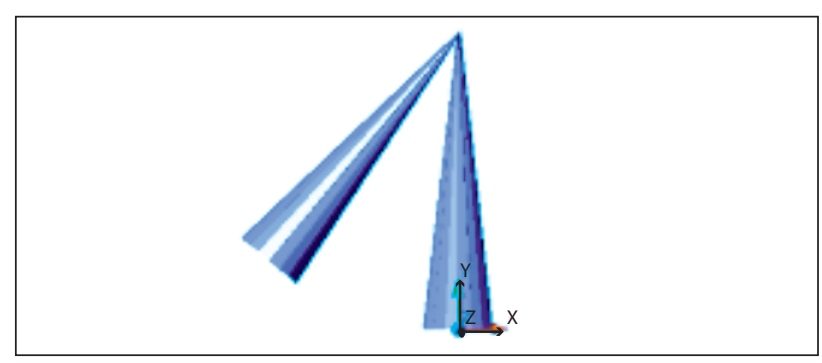

Figure 9. Folded configuration of the model.

The experiment is conducted under micro-gravity conditions, and the atmospheric pressure is maintained at $1.03 \mathrm{kPa}$. The tube has a section radius of $12 \mathrm{~mm}$ and a length of $200 \mathrm{~mm}$. The membrane has a thickness of $104 \mu \mathrm{m}$, an elastic modulus of $1.08 \times 10^{8} \mathrm{~N} / \mathrm{m}^{2}$, a Poisson's ratio of 0.3 and a density of $910 \mathrm{~kg} / \mathrm{m}^{3}$. The inflatable gas is nitrogen, the experimental temperature is $300.68 \mathrm{~K}$, and the gas constant is $296.798 \mathrm{~J} / \mathrm{kgK}$. The shell of the rigid column has a mass of $75.7 \mathrm{~g}$, a section radius of $5 \mathrm{~mm}$ and a thickness of $30 \mathrm{~mm}$.

The deployment mechanism of this tube model is simulated using the developed simulation software to obtain the configurations during the deployment process, which is illustrated in Fig. 10. The simulation results for the deployment process are in good agreement with the experimental results.

The internal chamber of the tube is divided into a bottom and a top compartment. The variations in the pressures in the two compartments with the deployment time are estimated using Eq. 9 and are shown in Fig. 11. Initially, the pressure in the bottom compartment increases, and the section between the two compartments is opened at a time of $0.9 \mathrm{~s}$. The gas then enters the top compartment, and the pressure increases. Finally, the pressure in the two compartments reaches the design pressure of $31 \mathrm{kPa}$.

Figure 12 shows that the folded angle between the two compartments changes with the deployment time. The folded angle is initially 40 degrees and increases after 0.9 s. Finally, the tube straightens, and the folded angle reaches 180 degrees.

The $\mathrm{x}$ - and $\mathrm{z}$-coordinates at the top end of the tube change during the deployment process and are shown in Fig. 13. The simulation and experimental results agree well with each other. 

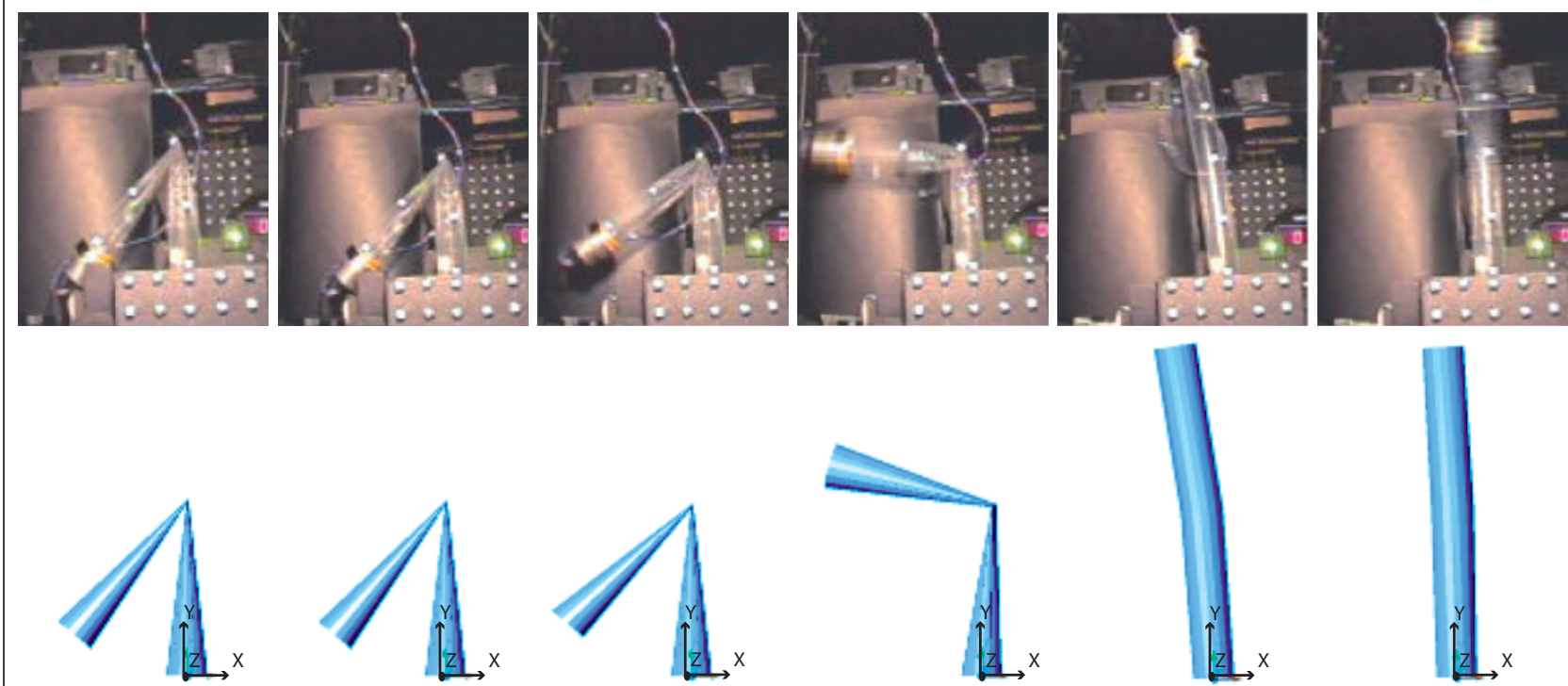

$0.4 \mathrm{~s}$

$0.6 s$

$1.0 \mathrm{~s}$

$1.3 \mathrm{~s}$

$1.9 \mathrm{~s}$

$2.3 \mathrm{~s}$

Figure 10. Comparison between experimental and simulation results.

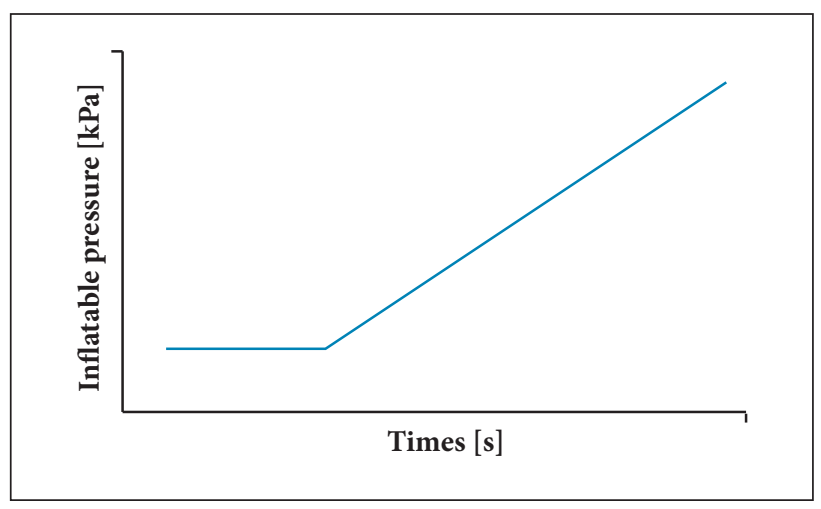

Figure 11. Changes in inflatable pressure.

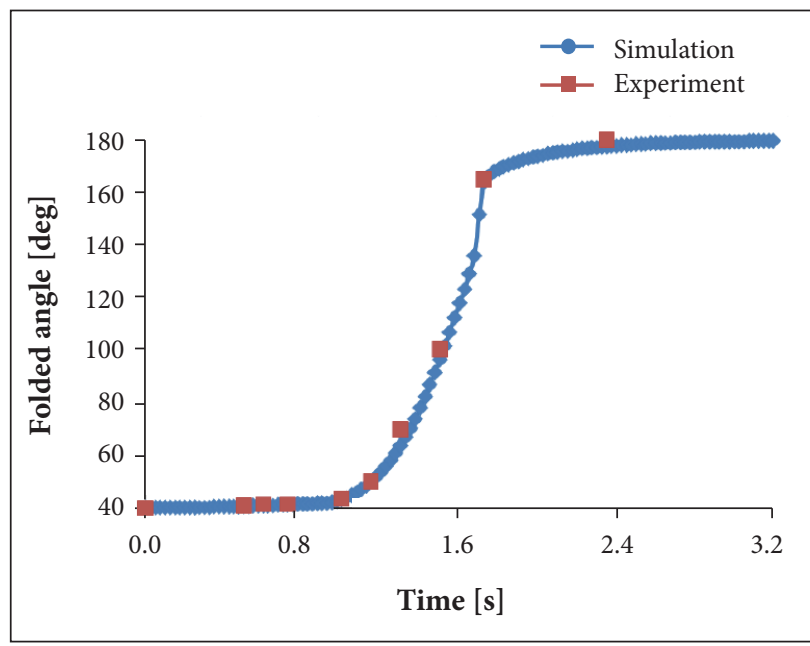

Figure 12. Folded angle between two compartments.

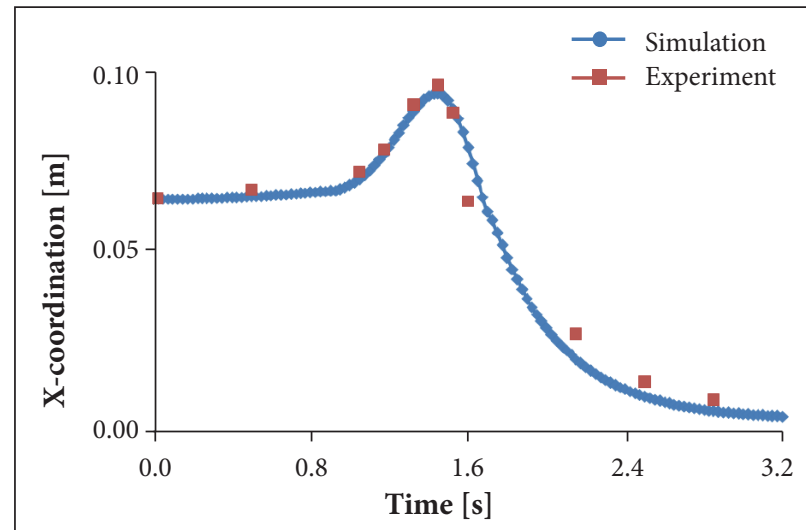

(a)

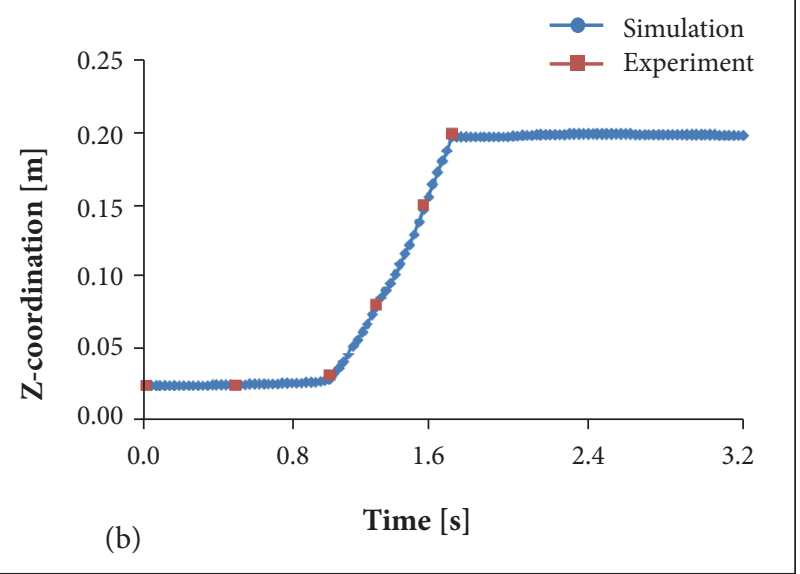

Figure 13. Coordinates at the top end of the tube. (a) X-coordinate; (b) Z-coordinate. 


\section{COMPLETE ANTENNA MODEL}

A complex inflatable antenna structure similar to that in the IAE is analyzed and modeled. The model includes inflatable tubes, an inflatable torus, a reflector and tension cables. The support structure for the inflatable antenna structure consists of three tubes and a torus. There are 24 tension cables between the reflector and the support structure. The geometric parameters of the unstressed configuration are given here. The caliber $D$ of the reflector is $6 \mathrm{~m}$, and the focus length $f$ is $7.5 \mathrm{~m}$. The same equation is used for the reflector surface as for the canopy. The length of a single tube is $8.0498 \mathrm{~m}$, and the section radius is $0.06 \mathrm{~m}$. The outer diameter of the torus is $7.5 \mathrm{~m}$, and the inner diameter is $7.0 \mathrm{~m}$. The section radius of the torus is $0.125 \mathrm{~m}$.

The membrane of the tubes, the torus and the reflector is made of Kapton. The membrane has an elastic modulus of 3.5 GPa, a Poisson's ratio of 0.35 , a thickness of $0.127 \mathrm{~mm}$, and a density of $1,450 \mathrm{~kg} / \mathrm{m}^{3}$. The tension cables are made of Kevlar and have a diameter of $1.2 \mathrm{~mm}$. The elastic modulus of Kevlar is $131 \mathrm{GPa}$. The torus has 288 elements and 144 nodes. The reflector has 720 elements and 362 nodes. Each tube has 48 elements and 30 nodes. Thus, the entire model has 1,152 elements and 596 nodes.

The reflector has a folded ratio of 0.5 in the longitudinal direction. The circumferential direction of the reflector and the torus are divided into 24 parts, i.e. the fold number $\mathrm{n}=24$. The radius of the torus axes curve in the folded configuration is $1.8 \mathrm{~m}$. The tube is divided into four parts lengthwise and is folded using a Z-fold pattern. The entire antenna is stowed into the volume of a cylinder with a radius of $2 \mathrm{~m}$ and a height of $2.6 \mathrm{~m}$. The design pressures of the torus and the tubes are $23.6 \mathrm{kPa}$, and the design pressure of the reflector is $100 \mathrm{~Pa}$. The tubes, torus and reflector have 4, 24 and 1 inflated compartments, respectively.

The bottom point of the three tubes is connected to the satellite and remains fixed during the deployment process.

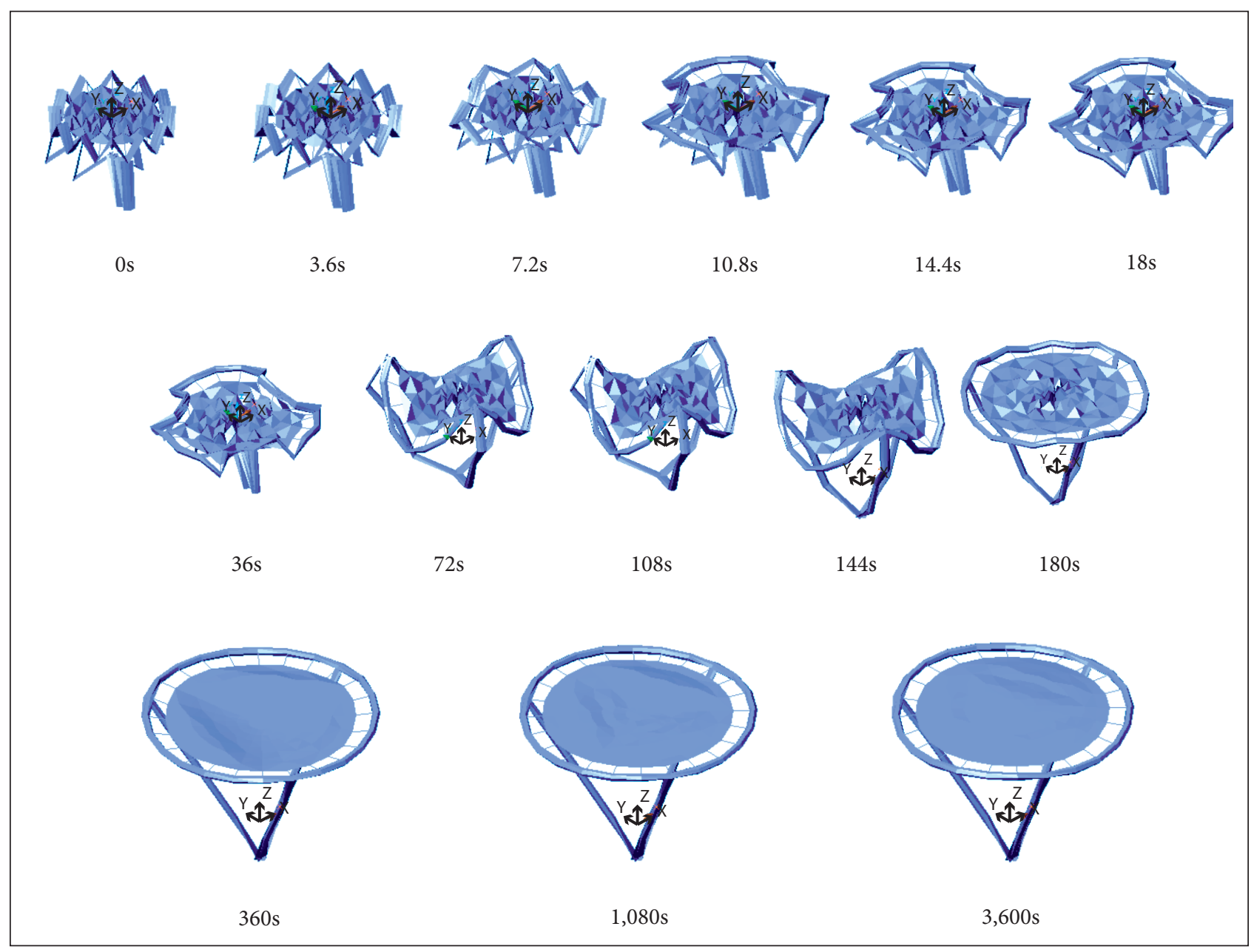

Figure 14. Individual deployment configurations. 
Several configurations during the deployment process are shown in Fig. 14. First, the tube and torus are inflated. The tube straightens, which causes the torus and the reflector to move along the positive $\mathrm{Z}$-coordinate. The cables cause the reflector to move. The axes of the torus tend to form a triangular shape, which is initially driven by the three tubes and then slowly deployed into a circle curve.

The results of the deployment dynamic simulation show that the three tubes and the axes of the torus are fully deployed at 360 s. The 3-D deployment velocities of the joint between the tube and the torus are shown in Fig. 15. At this time, wrinkles can be observed along the section direction of the torus. The reflector is pulled by the tension cables, but many wrinkled regions in the reflective surface remain. The reflector and the torus section continue to be deployed after $360 \mathrm{~s}$. The torus section is fully deployed at $1,080 \mathrm{~s}$. The reflector is slowly subjected to the design pressure and moves from its initial folded configuration to its final configuration. The internal force in the reflector surface is then transferred to the torus by the tension cables. The results show that the elastic deformation of the torus is very small.

The simulation results are used to evaluate the deployment dynamics of the antenna. The deployment process takes 3,600 s. The torus has a large diameter of $7.5052 \mathrm{~m}$ and a small diameter of $7.0855 \mathrm{~m}$. The length of each tube is $8.0429 \mathrm{~m}$. The fully deployed configuration has a high shape precision. Over the whole deployment process, the entire antenna structure moves smoothly from the folded configuration into the final design configuration. The results also validate the efficiency of the folded methods and the deployment mechanism simulation software of the inflatable structures.

\section{CONCLUSION}

Parametric model methods and simulation software for the deployment mechanism of inflatable antenna structures are investigated in this study. The unstressed and folded configurations of the entire structure are modeled parametrically. The time histories of the structural configuration during the deployment process and the dynamic parameters of each node are thus obtained by the simulation work. Numerical examples are used to validate the simulation software and the folded methods for inflatable structures. The results show that the entire antenna can be smoothly deployed from its initial folded

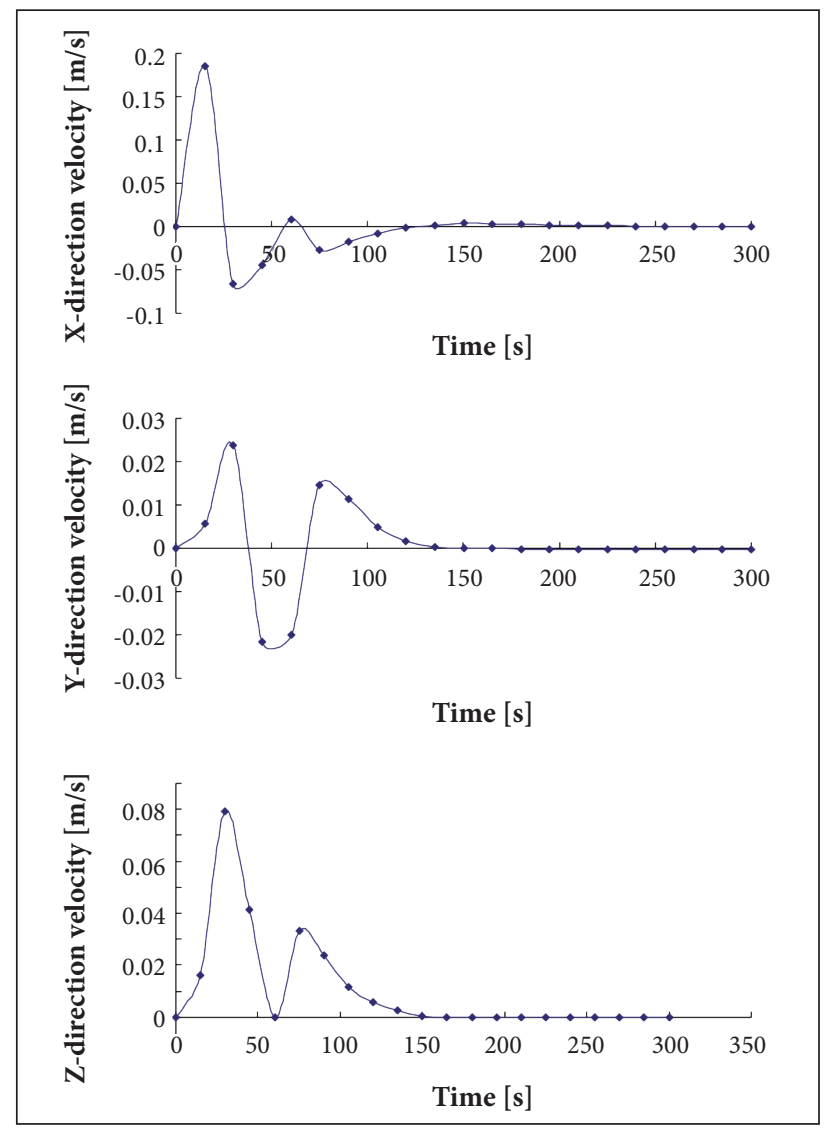

Figure 15. Deployment velocities of the joint between the tube and the torus.

configuration into the final fully deployed configuration. The shape of the fully deployed configuration is in good agreement with the design configuration.

In the present study, the number of elements used in the numerical model is not very large. The number of elements should be increased to verify the convergence of the numerical simulation. The primary members, such as the tubes and the torus, can be assembled to form complicated inflatable structures such as solar arrays and light shields. Assembly techniques for the parametric model design of the folded configuration should be studied in the future.

\section{ACKNOWLEDGEMENTS}

This work was supported by the National Natural Science Foundation of China (Grant No. 11402229, 51378458) and the Zhejiang Province Natural Science Foundation (Grant No. LQ14A020003, LY13E080002). 


\section{REFERENCES}

Bouzidi, R., Buytet, S. and Van, A.L., 2013, "A Numerical and Experimental Study of the Quasi-Static Deployment of Membrane Tubes", International Journal of Solids and Structures, Vol. 50, No. 1, pp. 651-661. doi: 10.1016/j.ijsolstr.2012.10.027

Capua, M.D., Akin, D.L. and Davis, K., 2011, "Design, Development, and Testing of an Inflatable Habitat Element for NASA Lunar Analogue Studies", AIAA-2011-5044, pp. 1-20.

Clem, A.L. and Smith, S.W., 2000, "A Pressurized Deployment Model for Inflatable Space Structures", AIAA-2000-1808, pp. 1-11.

Cliff, E.W. and Ron, C.S., 2001, "A Hybrid Inflatable Dish Antenna System for Spacecraft", AIAA-2001-1258, pp. 1-9.

David, L., 2003, "Inflatable Deployed Membrane Waveguide Array Antenna for Space", AIAA-2003-1649, pp. 1-7.

Fang, H.F., Liu, M.C. and Hah, J., 2006, "Deployment Study of a SelfRigidizable Inflatable Boom", Journal of Spacecraft and Rockets, Vol. 43, No. 1, pp. 25-30. doi: 10.2514/1.3283

Haug, E., Protard, J.B. and Gerren, A.M., 1991, "The Numerical Simulation of the Inflation Process of Space Rigidized Antenna Structure", Proceedings of the International Conference: Spacecraft Structures and Mechanical Testing, ESA, Zurich, Switzerland, pp. 862-868.

Katsumata, N., Natori, M.C. and Yamakawa, H., 2014, "Analysis of Dynamic Behavior of Inflatable Booms in Zigzag and Modified Zigzag Folding Patterns", Acta Astronautica, Vol. 93, No. 1, pp. 45-54. doi: 10.1016/j.actaastro.2013.06.008

Kramer, R.M.J., Cirak, F. and Pantano, C., 2013, "Fluid-Structure Interaction Simulations of a Tension-Cone Inflatable Aerodynamic Decelerator", AIAA Journal, Vol. 51, No. 7, pp. 1640-1656.
Lampani, L. and Gaudenzi, P., 2010, "Numerical Simulation of the Behavior of Inflatable Structures for Space", Acta Astronautica, Vol. 67, No. 3, pp. 362-368. doi: 10.1016/j.actaastro.2010.02.006

Miyazaki, Y. and Uchiki, M., 2002, "Deployment Dynamics of Inflatable Tube", AIAA-2002-1254, pp. 1-10.

Salama, M., Fang, H.F. and Lou, M.C., 2001, "Resistive Deployment of Inflatable Structures", AIAA-2001-1339, pp. 1-9.

Wang, C.C.L., Smith, S.S.F. and Yuen, M.M.F., 2002, "Surface Flattening Based on Energy Model", Computer-Aided Design, Vol. 34, No. 11, pp. 823-833.

Wang, J.T. and Johnson, A.R., 2002, "Deployment Simulation of Ultra-Lightweight Inflatable Structures", AIAA-2002-1261, pp. 1-14.

Wei, J.Z., Tan, H.F., Wang, W.Z. and Cao, X., 2015, "Deployable Dynamic Analysis and On-Orbit Experiment for Inflatable GravityGradient Boom", Advances in Space Research, Vol. 55, No. 2, pp. 639-646. doi: 10.1016/j.asr.2014.10.024

Xiao, X., Guan, F.L. and Xu, Y., 2010, "Numerical Analysis and Experimental Study on Deployment Process of Coil-Folded Inflatable Tube" (in Chinese), Journal of Zhejiang University (Engineering Science), Vol. 44, No. 1, pp. 184-189.

Xu, Y., Guan, F.L., Chen, J.J. and Zheng, Y., 2012, "Structural Design and Static Analysis of a Double-Ring Deployable Truss for Mesh Antennas", Acta Astronautica, Vol. 81, No. 2, pp. 545-554. doi: 10.1016/j.actaastro.2012.09.004

Xu, Y., Guan, F.L. and Ma, Y.H., 2007, "Reflector Design and Precision Measurement of Inflatable Antenna" (in Chinese), Journal of Zhejiang University (Engineering Science), Vol. 41, No. 11, pp. 1921-1926. 